tumor also showed a trend that was less dramatic toward an increased disease free survival time with chemotherapy. Irrespective of the therapeutic regimen, children less than 4 years of age fared worse than older children. In ependymomas, same responses to chemotherapy have been noted in recurrent tumors but a consistently effective drug program has not been identified. Malignant germ cell tumors are often given combination chemotherapy plus irradiation. Recently, chemotherapy has also been used for germinamas to reduce subsequent irradiation dosage. Chemotherapy has also been used along with radiotherapy for the treatment of pinealomas and pinealoma blastamas which usually do not respond well to radiotherapy alone. Acute leukemias (especially lymphoblastic leukemia, and non-Hodgkin lymphama) may involve the central nervous system in children. Non-Hodgkin lymphoma of the brain is being reported with increasing frequency in patients with AIDS and is projected to be one of the most common neurologic neoplasms within several years. Treatment of brain lymphoma has traditionally involved systemic chemotherapy, intrathecal chemotherapy, and irradiation. Patients with AIDS have difficulty tolerating large doses of chemotherapy and may benefit from antiretroviral and immomodulator drugs in future trials.

The long-term adverse effects of therapy of brain tumors have assumed greater importance since increasing numbers of children survive treatment programs. Approximately one-half may manifest significant intellectual or behavioral retardation, particularly in those under 3 years of age. White matter abnormalities, calcifications, and brain atrophy may be demonstrated on CT scans and MRI. Histopathologic findings after cranial irradiation have included demyelinization and mineralizing microangiopathy. Short stature resulting from growth hormone secretory dysfunction occurs in brain tumor patients who received radiation therapy and hypothyroidism may occur after craniospinal irradiation. (Kadota $\mathrm{R} \mathrm{P}$ et al. Brain tumors in children. $J$ Pediatr April 1989; 114:511-519).

COMMENT. An estimated 1200-1500 new cases of brain tumors will be diagnosed annually in American children less than 15 years of age. Campared with other childhood cancers, brain tumors have not been intensively studied by pediatric oncologists. With the recent advances in chemotherapy and the concerns regarding adverse effects of radiotherapy, oncologists are playing a larger role in the treatment of brain tumors in children. Well designed controlled studies in multiple collaborative centers will be necessary to prove the effectiveness of this form of therapy.

\title{
DYSPLASTIC GANGLIOCYTOMA AND PARTIAL SEIZURES
}

The clinical, radiologic and EEG features of three children with dysplastic gangliocytoma of the cerebral hemispheres and drug resistant partial seizures are described from the Comprehensive Epilepsy Center and Department of Neurology, Miami Children's Hospital, Miami, FL. A two year old girl had recurrent right-sided focal motor seizures that began within hours of birth; a 15 year old boy had habitual left-sided sensory seizures and infrequent grand mal attacks beginning at age three; and an eight month old boy was hospitalized following an episode of head trauma with unconsciousness and apnea followed by recurrent seizures consisting of staring, eye blinking and left versive head movement. None of the cases manifested a mass effect 
and the EEG findings lateralized to one hemisphere. Following cortical resection, two became seizure free and the third was almost seizure free. The authors consider that dysplastic gangliocytoma may be an important and surgically remedial cause of very early malignant partial seizures. (Duchowny M S et al. Dysplastic gangliocytoma and intractable partial seizures in childhood. Neurology April 1989; 39:602-604).

COMENT. Gangliocytomas are usually localized to the cerebellum and those originating in the cerebral hemispheres are much less cammon but may be epileptogenic. CT demonstrates hyperdense noncontrast enhancing regions and MRI's reveal medium intensity regions in the T-1 weighted and proton density images. The authors consider that subdural electrode recording can facilitate early surgical intervention by localizing epileptogenic regions and defining cortical regions of functional significance. The use of subdural electrodes to study the EEG features of simple partial seizures in seven patients is described in an additional article in this same issue of Neurology. (Devinsky $\mathrm{O}$ et al. Neurology April 1989; 39;527-533).

\section{LEARNING DISABILITIES}

READING WITH ONE HEMISPHERE

The reading performance of two right-handed teenage girls who had undergone hemispherectamy for intractable epilepsy was examined at the MRC Applied Psychology Unit, Cambridge, the Department of Developmental Pediatrics, Institute of Child Health and Department of Psychological Medicine, Hospital for Sick Children, and the Neurosurgical Unit, Maudsley Hospital, London. Both subjects had developed normal language and reading capacities before the onset of their illness. One patient, whose symptoms of left hemisphere abnormality comenced at the age of 13 years and whose left hemisphere was removed at the age of 15 , was poor at virtually all aspects of reading; but her pattern of reading performance was not one of undifferentiated impairment. She was essentially perfect at recognizing letters, although not very successful at naming them and totally unable to give their sound equivalents. She was reasonably good at discriminating very camon words from or thographically similar nonwords, but her lexical decision performance fell off quickly as word frequency declined. She could comprehend printed words as measured by matching to pictures, when those corresponded to common concrete objects but she occasionally made semantic errors in this task, and her success was dependent on maximal degrees of both word familiarity and concreteness. She also had some degree of success in oral reading of the most familiar and highly imageable words of the type that she could understand and had no means of translating a printed word into a phonological response except on the basis of its meaning. She could not read aloud nonsense words although she could repeat them perfectly. This patient's reading performance was identical in pattern though not in level to most cases of acquired deep dyslexia. There is a strong case for the equation of deep dyslexic reading with right hemisphere reading. The reading performance of the second patient whose right hemisphere had been removed was not as advanced in level as that of a normal 17 year old but it showed no abnormality in any subcomponent or reading skill. The discrepancy between her chronological and 